\title{
Diagnostic value of four coagulation function indicators for distinguishing between tuberculous pleural effusions and malignant pleural effusions due to lung adenocarcinoma
}

Rui cheng Li ( $\square$ lirc16@163.com )

Xi'an Tangdu Hospital of No4 Military Medical University https://orcid.org/0000-0003-4857-3716

Zhaowei Gao

Xi'an Tangdu Hospital of No4 Military Medical University

Fang Lin

Xi'an Tangdu Hospital of No4 Military Medical University

Xi Wang

Xi'an Tangdu Hospital of No4 Military Medical University

Bingquan Gu

Xi'an Tangdu Hospital of No4 Military Medical University

Yanni Fan

Xi'an Tangdu Hospital of No4 Military Medical University

Yanzhi Lu

Xi'an Tangdu Hospital of No4 Military Medical University

Congcong Ding

Xi'an Tangdu Hospital of No4 Military Medical University

Haihang Ma

Xi'an Tangdu Hospital of No4 Military Medical University

Ke Dong

Xi'an Tangdu Hospital of No4 Military Medical University

Huizhong Zhang

Xi'an Tangdu Hospital of No4 Military Medical University

\section{Research article}

Keywords: pleural effusion, coagulation indicators, tuberculosis, lung adenocarcinoma, receiver operating characteristic analysis

Posted Date: June 2nd, 2020 
DOI: https://doi.org/10.21203/rs.3.rs-31348/v1

License: (c) (i) This work is licensed under a Creative Commons Attribution 4.0 International License. Read Full License 


\section{Abstract}

Background: Coagulation function indicators are often used to evaluate patients' coagulation function.They are rarely used in disease diagnosis,their use is limited to somecoagulopathies. We described the pattern of coagulation function indicators in patients with pleural effusions and determined the diagnostic value of different abnormal patterns of these indicators for distinguishing tuberculous pleural effusions (TPEs) from malignant pleural effusions associated with lung adenocarcinoma (ADMPEs).

Methods: Patients with AD-MPEs or TPEs participated in this retrospective study.Coagulation functionindicators, including activated partial thromboplastin time, prothrombin time (PT), fibrinogen (FIB), thrombin time, fibrinogen degradation products (FDP), and D-dimer(D-D), were measured; their levels and abnormal patterns were analyzed separately and in combination,to determine the diagnostic performance of individual and combined values and the optimal diagnostic model.

Results: One hundred patients with AD-MPEs and 84 patients with TPEs participated.PT, FIB, FDP, and DDlevels in patients with TPE were significantly higher than those in patients with AD-MPE (all $P<0.01)$. Receiver operating characteristiccurves revealed that PT, FIB, FDP, and D-Dvalues, particularly FDP and D-D, could distinguish betweenAD-MPE and TPE. The diagnostic performance was better for the combination of the four indicators than for any single indicator (Youden's index: 0.513 ; area under the curve: 0.810 ). The proportion of patients with abnormal patterns of all four indicators was significantly higher in those with TPE than in those with AD-MPE $(P<0.001)$.

Conclusions: Thecombined values of PT, FIB, FDP, and D-Dhave diagnostic value for distinguishing TPE from AD-MPE.

\section{Background}

In China, the main causes of exudative pleural effusions are tuberculosis and lung adenocarcinoma [1]. Patients with lung adenocarcinoma often have pleural effusions, of which about $20 \%$ are the first sign of lung adenocarcinoma, and $30 \%$ to $40 \%$ develop pleural effusions in the course of the disease, which are indicative of a poor prognosis and short survival [2]. Tuberculous pleurisy is the main cause of pleural effusions in areas with a high prevalence of tuberculosis [3]. Although there are some clinical similarities between pleural effusions associated with lung adenocarcinoma (AD-MPE) and tuberculous pleural effusions (TPE), the diagnosis, treatment and prognosis of the two types of effusion are quite different. The similarities between AD-MPE and TPE,their delayed onset, and invasiveness of the pathology pose a major challenge in the differential diagnosis of these two conditions [4].

In recent years, some novel markers have been reported for the differential diagnosis of pleural effusions, but few have been successfully applied in clinical practice [5-7]. Coagulation function can be assessed using a very basic test and is often tested in patients admitted to hospital.Coagulation function testing is often used to evaluate the coagulation function of patients undergoing surgery and for the monitoring of 
anticoagulant therapy [8-11]. It is rarely used in disease diagnosis, and when it is, its use is limited to diagnosing certain coagulo pathies [12]. To date, there have been no studies of the levels and the patterns of coagulation function indicators for distinguishing patients with TPE from AD-MPE. The objectives of this study were to describe the pattern of coagulation function indicators in patients with pleural effusions, and to determine the diagnostic value of different abnormal patterns of these indicators for distinguishing TPE from AD-MPE.

\section{Materials And Methods}

\section{Patients}

We conducted a retrospective analysis of data from patients diagnosed with pleural effusions at the Tangdu Hospital, Fourth Military Medical University from September 2017 to December 2018. All patients had a diagnostic work-up and the cause of the PE was based on a combination of clinical findings, histopathology, cytology, or empirical diagnosis(response to therapy).

In order to be eligible for inclusion in the analysis, all patients had to satisfy the following conditions: 1 ) no bleeding tendency, hematopoietic dysfunction, or taking anticoagulant drugs, no anti-tumor or antituberculosis treatment prior to admission; 2) The clinical data were complete; 3) have no obvious abnormalities in heart, liver or kidney function; 4) no other tumors apart from lung adenocarcinoma.

This study was approved by the Ethics Committee of Tangdu Hospital, Fourth Military Medical University (TDLL-201709-24), and informed consent was waived on the grounds that it was a retrospective study.

\section{Collection and detection of plasma samples}

Prior to any cancer and tuberculosis-directed therapy, a sample of $2 \mathrm{ml}$ fasting venous bloodwascollected from each patient in a $2 \mathrm{ml}$ tube containing $0.109 \mathrm{~mol} / \mathrm{L}$ sodium citrate at a ratio of one-part citrate to nine parts of blood, and centrifuged for 10 minutes at $2500 \mathrm{~g}$.

Indicators of coagulation function were measured using the turbidimetry assay (SYSMEX, Kobe, Japan). These measures included activated partial thromboplastin time (APTT), prothrombin time (PT), fibrinogen content (FIB), thrombin time (TT), fibrinogen degradation products (FDP) and D-dimer (D-D). The normal ranges of the 6 indicators were as follows: APTT $21.1-36.5 \mathrm{~s}$; PT 9.8-12.1 s; FIB 1.8-3.5 g/L; TT 14-21 s; FDP 0-5 $\mu \mathrm{g} / \mathrm{mL}$; D-D 0-1 $\mu \mathrm{g} / \mathrm{mL}$.

\section{Statistical analysis}

Data was analyzed using SPSS Version 18.0 statistical software (IBM, Armonk, NY, USA). Patients were assigned to two groups according to whether they were diagnosed with AD-MPE or TPE. AD-MPE was used as the reference condition in comparisons. The indicators with values that followed a normal distribution (APTT, PT, FIB, TT) were compared directly using the independent Student's t-test. FDP and D$D$ values were log-transformed values and the log values were compared using the Student's t-test. 
Indicators that differed between the two groups were analysed further using receiver operating characteristic (ROC) curves. ROC curves were plotted for each coagulation indicator, and for the indicators combined to determine the optimal cut-point values. Values of each coagulation indicator were classified as either normal or elevated based on the cut-points determined using the ROC analysis. The optimal cut-off value was determined at the maximum value for Youden's index (YI). The categorical values of the coagulation indicators in the patients with AD-MPE and TPE were compared using chi square tests. P-values $<0.05$ were regarded as statistically significant.

\section{Results}

Comparison of basic characteristics and coagulation indicators in patients with pleural effusions due to lung adenocarcinoma and tuberculosis

Data from 184 patients, 100 with AD-MPE and 84 with TBE, were used in the analysis. Table 1 and Figure 1 show comparisons of basic patient characteristics and the levels of the coagulation indicators according to the group. There were no statistically significant differences in sex, age, blood pressure, body mass index or TT in patients with TPE and AD-MPE. The levels of PT, FIB, logFDP and logD-D were significantly higher in patients with TPE than in those with AD-MPE (Figs. 1(A) - (D)), especially logFDP and $\log \mathrm{D}-\mathrm{D}$.

Clinical diagnostic role of abnormal patterns for coagulation function indicators in patients with tuberculous pleural effusion

There was variation in the patterns of coagulation indicator abnormalities between the two groups (Table 2 and Fig. 2). Among patients with TPE, the most common patterns of abnormality were elevations in PT, FIB, FDP and D-D (44.1\%), or elevations in PT, FDP and D-D (22.6\%). However, among patients with ADMPE, the proportion of the two abnormal patterns was both $10.0 \%$.

The value of four coagulation function indicators for distinguishing between malignant pleural effusions due to lung adenocarcinoma from and tuberculous pleural effusions

In order to further clarify the value of PT, FIB, FDP and D-D in the differential diagnosis of AD-MPE and $T P E$, a single index was developed based on and combination of these four indexes were analyzed by ROC curve (Table 3, Figure 3). For PT, FIB, FDP and D-D, using AD-MPE as a reference, the optimal discrimination of TPE was determined at cut-off value of $11.65 \mathrm{~s}, 4.69 \mathrm{~g} / \mathrm{L}, 0.69 \mu \mathrm{g} / \mathrm{mL}$ and $0.25 \mu \mathrm{g} / \mathrm{mL}$, respectively. Of the four indicators, FIB has the highest sensitivity and the lowest specificity and YI,while FDP had the highest specificity and the lowest sensitivity; D-D had the highest YI and AUC; and the YI of each of the four indicators were $<0$.5.When PT, FIB, FDP and D-D were used in combination and presented by ROC curve, the diagnostic performance was improved (Table 3).

\section{Discussion}


Using blood and PE markers is useful for distinguishing TPEs from AD-MPEs, such as adenosine deaminase and carcinoembryonic antigen $[13,14]$. A number of markers can be used to predict TPEs and AD-MPEs, although their use of markers is considerably less specific than histology. However, the results can complement each other [15]. As a routine diagnostic work-up, PT, FIB, FDP and D-D are often used to manage coagulation and fibrinolysis in patients; however, this is the first study to utilize coagulation indicators to distinguish TPEs from AD-MPEs.

Many studies on coagulation function and tumors have found that coagulation indicators, especially FIB, FDP and D-D, are useful for predicting the risk of occurrence, progression, metastasis, and prognosis of lung cancer: Piccioli [16] found that the probability of thromboembolism in patients with tumors was close to $10 \%$; Aminian et al. [17] found that the hypercoagulable state of tumor patients was closely related to cancer progression and prognosis; Palumbo et al. [18] found that FIB and platelets can increasethe possibility of tumor invasion by impeding natural killer cell-mediated elimination of tumor cells during tumor progression. However, there are few reports on the role of coagulation indicators in individuals with tuberculosis $[19,20]$, and there are even fewer reports on coagulation indicators in relation to TPE [21].Therefore, at the outset of this study, we hypothesized that the severity of coagulation dysfunction in patients with AD-MPE swould be more marked than in those TPEs. However, the result was the opposite of what we expected.This study found that the levels of APTT,PT,FIB,FDP and D-Dwere higher, and abnormal patterns were more prevalent in patients with TPEs than in those withAD-MPEs. The results suggest that the severity of coagulation dysfunction is more marked in individuals with benign TPEs than in those with AD-MPEs.

It has been reported that tissue factors are activated and released during acute infection of tuberculosis [22]. As the initiating factor of the endogenous coagulation pathway, tissue factor plays a very important role. PT is the primary indicator with which to monitor the endogenous coagulation pathway [23]. However,there have been no reports on the application of PT to monitor the changes of coagulation function in patients with TPE. In this study, it was found that $>70 \%$ of the patients with TPE had a high PT. whereas $<50 \%$ of patients with AD-MPE had a high PT, and this difference was statistically significant. This result differs from the consensus perception that lung cancer, especially lung adenocarcinoma, is more likely to cause coagulation dysfunction [24]. Therefore. in clinical practice, attention should be paid not only to FDP and D-D, but also to PT when acute tuberculosis is suspected.

When Mycobacterium tuberculosis invades the pleura, it activates the monocyte macrophage system and the bacteria are swallowed and degraded by macrophages. The degradation products induce a strong specific allergic reaction and destroy vascular endothelial cells [25]. Progress of lung adenocarcinoma leads to the destruction and disintegration of the tissue, resulting in non-specific inflammatory reactions and inflammatory mediators. The continuous inflammatory state constitutes the microenvironment of tumor progress [26]. About $90 \%$ of patients with malignant tumors have vascular endothelial cell injury, and this is most marked in people with lung adenocarcinoma [27]. The damage to vascular endothelium caused by pleural effusions can destroy the balance of the coagulation and fibrinolysis systems. For this reason, this study considered all possible abnormal patterns of coagulation function indicators in the two 
groups, and ten patterns of abnormal coagulation function caused by AD-MPE and TPE, which not only confirmed the results of previous studies, but also reflected the complexity of the two conditions. In this study, simultaneous abnormalities of PT, FIB, FDP and D-D,and PT, FDP and D-D, were present in 44\% and $23 \%$ of patients with TPE, which was higher than the $10 \%$ of these abnormalities in patients with AD$\operatorname{MPE}(P<0.05)$. These results indicate that clinicians should consider the possibility of TPE in patients with pleural effusions who have either of these two abnormal patterns of coagulation function indicators.

As degradation products of cross-linked fibrin, FDP and D-D are considered to be general indicators of coagulation activation and fibrinolysis [28-30]. In this study, FDP and D-D were the two indicators with the highest diagnostic performance, and FIB had the highest sensitivity, but their YIs, which are based on the sensitivity and specificity of the test were less than 0.5 . Therefore, in order to improve the power of these indicators to distinguish between AD-MPE and TPE, we combined PT, FIB, FDP and D-D to create a joint indicator.ROC curve analysis showed that the diagnostic performance of the combined indicator was better than that of any of the indicators alone. Therefore, although the determination of coagulation function is the most basic method for the differential diagnosis of AD-MPE and TPE, it had the characteristics of simplicity, economy, rapidity and is non-invasive, and is suitable for use in limitedresource settings and in community-based health facilities as an ideal way to distinguish TPE from ADMPE.

The primary limitation of this study was that participation was restricted to patients withAD-MPEsor TPEs, and patients with lung cancer and tuberculosis without pleural effusions were excluded.We are planning to conduct a further study with a larger sample size that takes into account the pathological classification of lung cancer, incorporates more types of tuberculosis, and reduces bias as far as possible, to further explore the value of using coagulation indicators in different pathological types of lung cancer and different types of tuberculosis.

\section{Conclusions}

Coagulation function indicators, especially if considered in combination, had relatively good sensitivity and specificity for distinguishing between pleural effusions due to lung adenocarcinoma and those due to tuberculosis. As measuring coagulation function indicators is a routine test, this method may be applied to the differential diagnosis of pleural effusions in community care settings.

\section{Abbreviations}

AD-MPE (malignant pleural effusion associated with lung adenocarcinoma)

APTT (partial thromboplastin time)

AUC (area under the curve)

DD ( D-dimer) 
FDP ( fibrinogen degradation product)

FIB ( fibrinogen)

PE (plural effusion)

PT ( prothrombin time)

ROC ( receiver operating characteristic)

TPE ( tuberculous pleural effusion)

TT ( thrombin time)

YI (Youden's index)

\section{Declarations}

\section{Ethics approval and consent to participate}

This study was approved by the Ethics Committee of Tangdu Hospital, Fourth Military Medical University.

\section{Consent for publication}

Not applicable.

\section{Availability of data and materials}

The datasets used during the current study available from the corresponding author on reasonable request.

\section{Competing interests}

The authors declare that they have no competing interests.

\section{Funding}

This work was supported by the Natural Science Foundation of China (no.81772485 and no. 81702732). This funding body had no role in the design of the study and collection, analysis, and interpretation of data and in writing the manuscript.

\section{Authors' contributions}

Conception and design: Ruicheng Li, Ke Dong and Huizhong Zhang. Experiments performance: Zhaowei Gao, Congcong Ding and Haihang Ma. Statistical analysis: Fang Lin, Yanni Fan and XiWang. Supervision: 
Bingquan Gu, Ke Dong and Yanzhi Lu. Article drafting: Ruicheng Li and Hui-zhong Zhang. All authors reviewed the manuscript.

\section{Acknowledgements}

None.

\section{References}

1. Hawatmeh A, Thawabi M, Jmeian A, Shaaban H, Shamoon F. Amiodarone-induced loculated pleural effusion without pulmonary parenchymal involvement: A case report and literature review. J Nat Sci Biol Med. 2017;8(1):130-133.

2. Siegel RL, Miller KD, Jemal A. Cancer statistics, 2016. CA Cancer J Clin. 2016;66(1):7-30.

3. Liu Y, Ou Q, Zheng J, et al. A combination of the QuantiFERON-TB Gold In-Tube assay and the detection of adenosine deaminase improves the diagnosis of tuberculous pleural effusion. Emerg Microbes Infect. 2016;5(8):e83.

4. Lama A, Ferreiro L, Golpe A, et al. Characteristics of Patients with Lymphangioleiomyomatosis and Pleural Effusion: A Systematic Review. Respiration. 2016;91(3):256-264.

5. Chen KY, Feng PH, Chang CC, et al. Novel biomarker analysis of pleural effusion enhances differentiation of tuberculous from malignant pleural effusion. Int J Gen Med. 2016;9:183-189.

6. Gonzalez A, Fielli M, Ceccato A, Luna C. Score for Differentiating Pleural Tuberculosis from Malignant Effusion. Med Sci (Basel). 2019;7(3).

7. Li C, Hou L, Sharma BY, et al. Developing a new intelligent system for the diagnosis of tuberculous pleural effusion. Comput Methods Programs Biomed. 2018;153:211-225.

8. Agren A, Holmstrom M, Schmidt DE, Hosokawa K, Blomback M, Hjemdahl P. Monitoring of coagulation factor therapy in patients with von Willebrand disease type 3 using a microchip flow chamber system. Thromb Haemost. 2017;117(1):75-85.

9. Larsen JB, Hvas AM. Predictive Value of Whole Blood and Plasma Coagulation Tests for Intra- and Postoperative Bleeding Risk: A Systematic Review. Semin Thromb Hemost. 2017;43(7):772-805.

10. Ranucci M. Bank blood shortage, transfusion containment and viscoelastic point-of-care coagulation testing in cardiac surgery. Br J Anaesth. 2017;118(6):814-815.

11. Schott U. Prehospital coagulation monitoring of resuscitation with point-of-care devices. Shock. 2014;41 Suppl 1:26-29.

12. Boender J, Kruip MJ, Leebeek FW. A diagnostic approach to mild bleeding disorders. J Thromb Haemost. 2016;14(8):1507-1516.

13. Lee SH, Park MJ, Choi SI, Lee EJ, Lee SY, In KH. Reactive oxygen species modulator 1 (Romo1) as a novel diagnostic marker for lung cancer-related malignant effusion. Medicine. 2017;96(4).

14. Liu YC, Lee SSJ, Chen YS, Tu HZ, Chen BC, Huang TS. Differential diagnosis of tuberculous and malignant pleurisy using pleural fluid adenosine deaminase and interferon gamma in Taiwan. $J$ 
Microbiol Immunol. 2011;44(2):88-94.

15. Assawasaksakul T, Boonsarngsuk V, Incharoen P. A comparative study of conventional cytology and cell block method in the diagnosis of pleural effusion. J Thorac Dis. 2017;9(9):3161-3167.

16. Piccioli A, Lensing AW, Prins $\mathrm{MH}$, et al. Extensive screening for occult malignant disease in idiopathic venous thromboembolism: a prospective randomized clinical trial. J Thromb Haemost. 2004;2(6):884-889.

17. Aminian A, Karimian F, Mirsharifi R, et al. Significance of platelet count in esophageal carcinomas. Saudi J Gastroenterol. 2011;17(2):134-137.

18. Palumbo JS, Talmage KE, Massari JV, et al. Platelets and fibrin(ogen) increase metastatic potential by impeding natural killer cell-mediated elimination of tumor cells. Blood. 2005;105(1):178-185.

19. Kager LM, Blok DC, Lede IO, et al. Pulmonary tuberculosis induces a systemic hypercoagulable state. J Infect. 2015;70(4):324-334.

20. Venkatasubramanian S, Tripathi D, Tucker T, et al. Tissue factor expression by myeloid cells contributes to protective immune response against Mycobacterium tuberculosis infection. Eur $\mathrm{J}$ Immunol. 2016;46(2):464-479.

21. Wang JY, Hsueh PR, Lee LN, et al. Mycobacterium tuberculosis inducing disseminated intravascular coagulation. Thromb Haemost. 2005;93(4):729-734.

22. Caccamo N, Dieli F. Inflammation and the coagulation system in tuberculosis: Tissue Factor leads the dance. Eur J Immunol. 2016;46(2):303-306.

23. Suzuki S, Morishima Y, Takita A, et al. Responses of prothrombin time and activated partial thromboplastin time to edoxaban in Japanese patients with non-valvular atrial fibrillation: characteristics of representative reagents in Japan (CVI ARO 7). Heart Vessels. 2019;34(12):20112020.

24. Nichols L, Saunders R, Knollmann FD. Causes of death of patients with lung cancer. Arch Pathol Lab Med. 2012;136(12):1552-1557.

25. Belay M, Bjune G, Ameni G, Abebe F. Diagnostic and treatment delay among Tuberculosis patients in Afar Region, Ethiopia: A cross-sectional study. Bmc Public Health. 2012;12.

26. lyengar NM, Hudis CA, Dannenberg AJ. Obesity and inflammation: new insights into breast cancer development and progression. American Society of Clinical Oncology educational book American Society of Clinical Oncology Annual Meeting. 2013:46-51.

27. Sorensen HT, Mellemkjaer L, Olsen JH, Baron JA. Prognosis of cancers associated with venous thromboembolism. N Engl J Med. 2000;343(25):1846-1850.

28. Aleman C, Alegre J, Monasterio J, et al. Association between inflammatory mediators and the fibrinolysis system in infectious pleural effusions. Clinical science (London, England : 1979). 2003;105(5):601-607.

29. Moresco RN, Junior RH, Claudio Rosa Vargas L, Mariano da Rocha Silla L. Association between plasma levels of D-dimer and fibrinogen/fibrin degradation products (FDP) for exclusion of 
thromboembolic disorders. Journal of thrombosis and thrombolysis. 2006;21(2):199-202.

30. Psathakis K, Calderon-Osuna E, Romero-Romero B, Martin-Juan J, Romero-Falcon A, RodriguezPanadero $F$. The neutrophilic and fibrinolytic response to talc can predict the outcome of pleurodesis. The European respiratory journal. 2006;27(4):817-821.

\section{Figures}
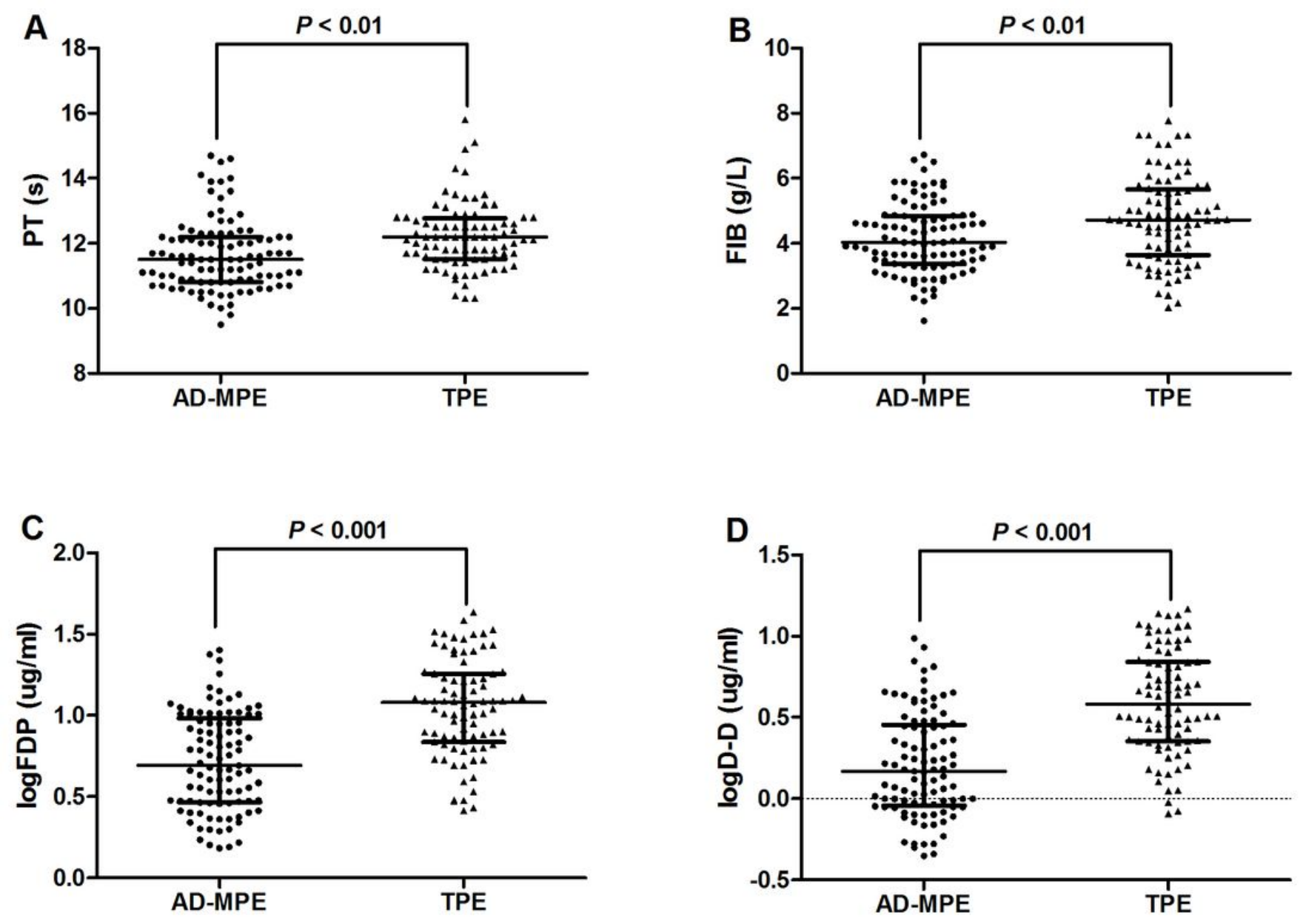

Figure 1

Comparison of each indicator according to the type of pulmonary effusion The levels of PT (A), FIB (B), FDP (C) and D-D (D) were significantly higher in patients with TPEs than in patients with AD-MPEs(all P< 0.01). $\log =$ logarithm of a corresponding indicator. Abbreviations: AD-MPE, malignant pleural effusion associated with lung adenocarcinoma; D-D, D-dimer; FDP, fibrin degradation product; FIB, fibrinogen;PT, prothrombin time; TPE, tuberculous pleural effusion 


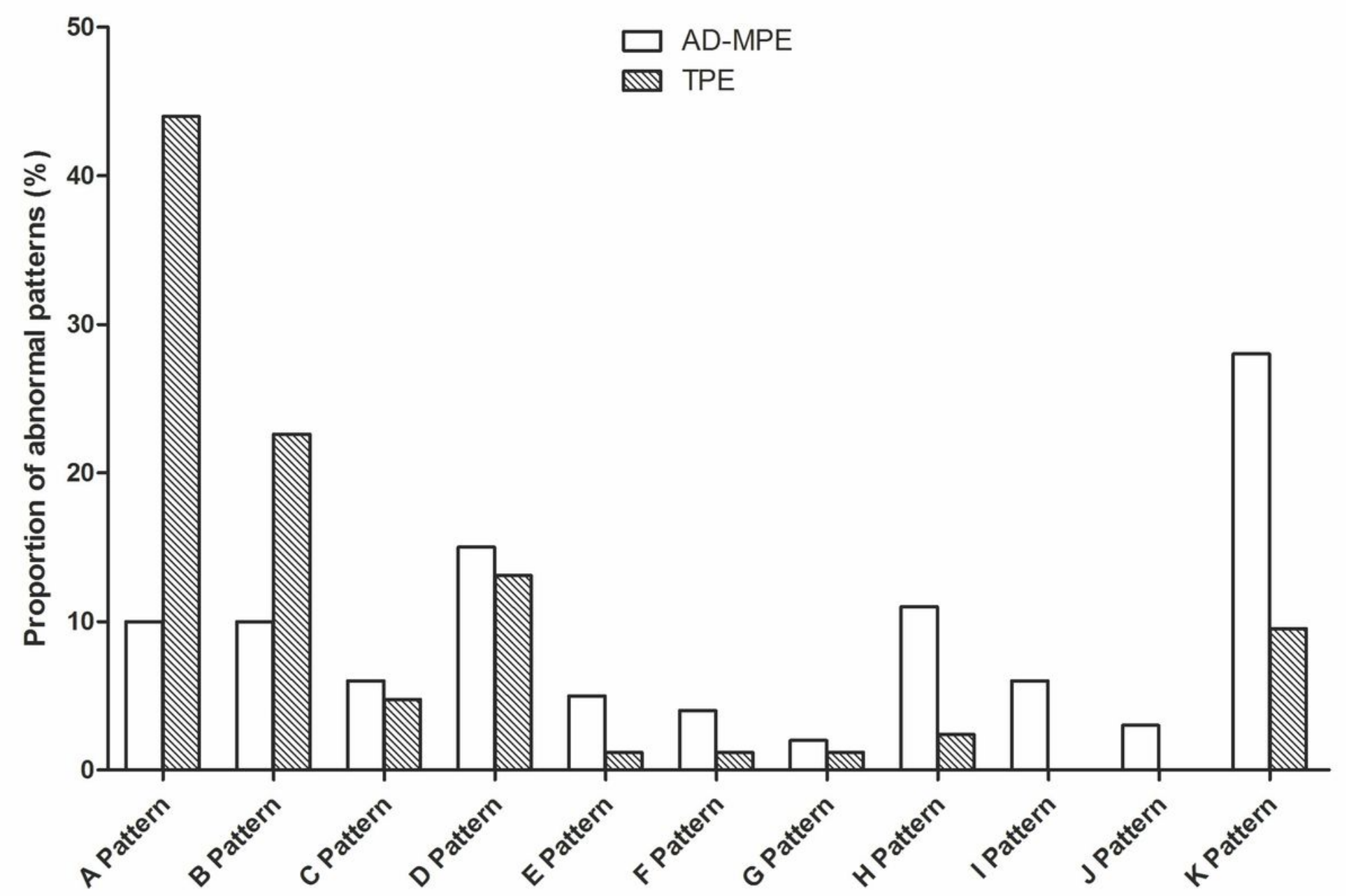

Figure 2

The prevalence of different abnormal patterns of coagulation function indicators according to the type of pleural effusion Pattern types: A: $\uparrow$ PT, FIB, FDP, D-D; B: $\uparrow$ PT, FDP, D-D; C: $\uparrow$ FIB, FDP, D-D; D: $\uparrow$ FDP, D-D; E: $\uparrow \mathrm{PT}, \mathrm{FDP} ; \mathrm{F}: \uparrow \mathrm{PT}, \mathrm{FIB}, \mathrm{FDP} ; \mathrm{G}: \uparrow \mathrm{FDP} ; \mathrm{H}: \uparrow \mathrm{PT} ; \mathrm{I}: \uparrow \mathrm{FIB} ; \mathrm{J}: \uparrow \mathrm{PT}, \mathrm{FIB}, \mathrm{TT}, \mathrm{FDP}, \mathrm{D}-\mathrm{D} ; \mathrm{K}: \uparrow$ TT, FDP, D-D There were statistically significant differences between the two groups for the abnormal patterns of $A$ and $B$ ( $A$ pattern, $P<0.001$; $B$ pattern, $P<0.05)$. Abbreviations: $\uparrow$, high; AD-MPE, malignant pleural effusion associated with lung adenocarcinoma; APTT, activated partial thromboplastin time; BMI, body mass index; D-D, D-dimer; FDP, fibrinogen degradation product; FIB, fibrinogen; PT, prothrombin time; SD, standard deviation; TPE, tuberculous pleural effusion; TT, thrombin time 


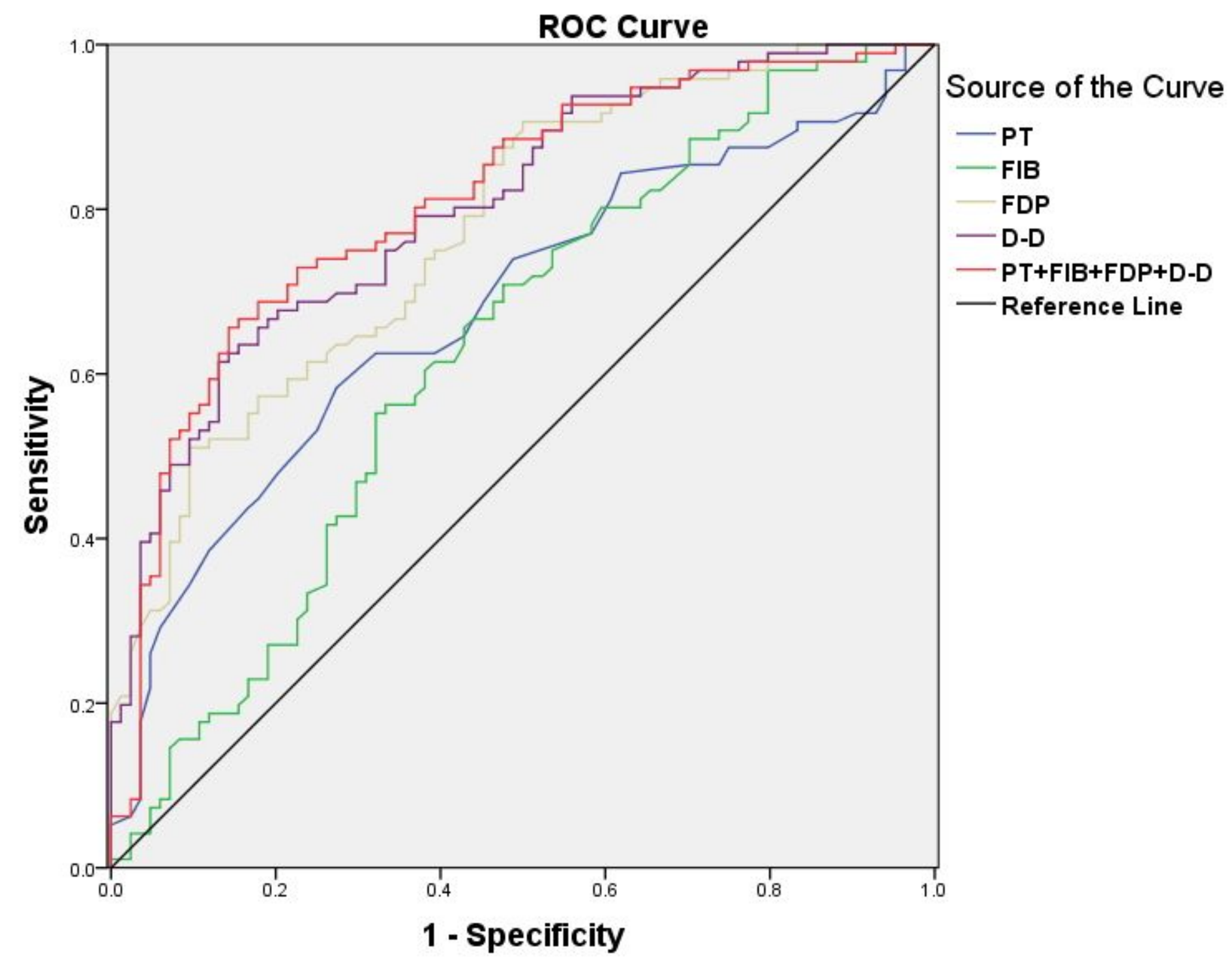

Figure 3

The area under the curve of the four indicators for distinguishing tuberculous pleural effusions from malignant pleural effusions due to lung adenocarcinoma Abbreviations: D-D, D-dimer; FIB, fibrinogen; FDP, fibrinogen degradation product; $\mathrm{PT}$, prothrombin time; ROC, receiver operating characteristic 